\title{
Experimental Study on the Wind Stability of Tongtai Bridge
}

\author{
a.C. XUN, ${ }^{b} \mathrm{Q}$. Zhao, ${ }^{\mathrm{C}} \mathrm{H} . J \mathrm{Gao}$ \\ ${ }^{a}$ Key Laboratory for Bridge and Tunnel of Shaanxi Province, Chang'an University, Xi'an 710064, \\ China \\ ${ }^{b}$ Management of Urban Expressway ZhangJiaKou, ZhangJiaKou 075000, China \\ ${ }^{c}$ Songjiang Tianjin Limited by Share Ltd, Tian'Jin 300221, China
}

KEYWORD: Curved Skew Arch Bridge; the Wind Stability; Wind Tunnel Test; Aeroelastic Model; Stability Analysis

ABSTRACT: For oblique arch, curved beam, space oblique composite system of suspension bridge, whose force transmission path and spatial stress is complex, robustness characteristics is strong, and no engineering experience can be used for reference. In order to analyse wind resistant performance of the Tongtai bridge, the aeroelastic model of the bridge is designed and fabricated by accurately simulating the flow field in the boundary layer of the field and using wind tunnel test technique, calculated vortex vibration, buffeting and aerodynamic stability of the arch-rib and whole bridge under uniform flow field and turbulent flow field at different attack angle, angle condition. The experimental results show that the bridge has good aerodynamic stability and no obvious vortex vibration occurs. The vertical and lateral displacement of the arch rib 1/4 section is higher than that of the crown, but it meets the requirement of wind resistance.

\section{INTRODUCTION}

In the 21 st century, the large span of the bridge structure system development by leaps and bounds, at the same time by the terrain, topography and shape and other aspects of the conditions of the restrictions, the bridge structure form also tends to diversity and complexity. Wind load becomes the control load of large span bridge structure and spatial structure system gradually ${ }^{[1][2]}$.After the accident of the wind tower bridge, the bridge wind engineering research get rapid development ${ }^{[3]}$.But most of the research on wind resistant safety is mostly focused on the suspension bridge and cable stayed bridge ${ }^{[4][5]}$. The wind tunnel test and numerical simulation as the main means of wind resistant design of structures, but due to the actual helical to cross sling combination system bridge less, the wind resistance capability study on this system is relatively blank. This paper has chosen the skew arch, curved beam oblique to cross sling combination bridge-Tongtai bridge as the research object, through wind tunnel tests to study corresponding characteristics of this kind of bridge wind load.

\section{ENGINEERING SURVEY}

As the standard of construction of Zhangjiakou City, Tongtai bridge is located in Zhangjiakou City Expressway north central line, across the Qingshui River, which is one of the connecting bridge of East Central and West. The steel girder, whose span is $190 \mathrm{~m}$ and section is a flat box single room 5 steel box girder, height is $3 \mathrm{~m}$ (center line), width is $34.144 \mathrm{~m}$, is located on the plane curve of $600 \mathrm{~m}$, and longitudinal slope is not located on the bridge deck, and the cross slope of the bidirectional $2 \%$ is provided. The main bridge are arranged at both ends of the limiting device, and a concrete counterweight. Arch rib is single box single room steel box arch rib, width is $7.04 \mathrm{~m}$, height is $3.8 \mathrm{~m}$, and the arch foot spacing is $180 \mathrm{~m}$, rise span ratio is 0.3451 , arch rib oblique span steel girder.

The bridge uses 28 slings, which are high strength galvanized steel wire and whose standard strength is $1670 \mathrm{MPa}$. At home and abroad, only 2 similar structural systems are built, but the maximum span is not more than 130m. The picture of Tongtai bridge is shown in Fig.1. 


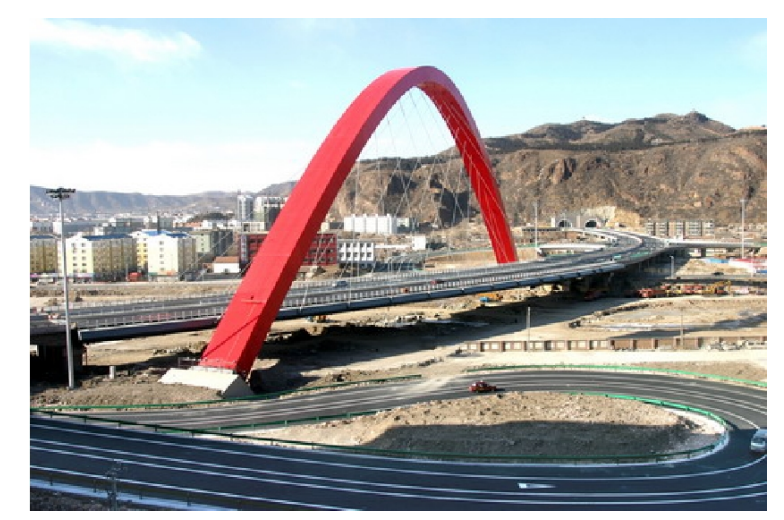

Figure 1. The completion drawing of Tongtai bridge

\section{MODEL DESIGN}

\section{MODEL DESIGN PRINCIPLES}

In the design of the full bridge aeroelastic model, besides the similar condition of the geometrical shape, the consistency condition of the elastic parameters, the inertia parameters, the gravity parameters, the viscous parameters and the damping parameters are also required ${ }^{[6][7]}$.Similar requirements of each component of the model design of the Tongtai bridge are shown in Table 1.

Table 1. Similar requirements for each component in the aeroelastic model design

\begin{tabular}{|c|c|c|c|}
\hline \multirow[t]{2}{*}{ component } & shape & Stiffness & \multirow[t]{2}{*}{ Aerodynamic } \\
\hline & & $\mathrm{EI}_{\mathrm{x}} \mathrm{EI}_{\mathrm{y}} \quad \mathrm{GJ}$ & \\
\hline $\begin{array}{l}\text { Main Beam } \\
\text { Sling } \\
\text { Arch Rib }\end{array}$ & $\square$ & $\begin{array}{lll}\square & \square \\
\square & \square\end{array}$ & $\square$ \\
\hline
\end{tabular}

The main span of the bridge is $190 \mathrm{~m}$. Taking into account the actual conditions of the wind tunnel, the model scale factor is identified as $C_{L}=1 / 66$, and considering the consistency condition of Froude number in the design model, the wind velocity ratio is identified as $C_{V}=1: \sqrt{66}=1: 8.12$, the frequency ratio is identified as $C_{f}=\sqrt{66}: 1=8.12: 1$. According to the above similarity principle, the corresponding similarity coefficient can be deduced, which are derived from the scale similarity coefficients.

\section{COMPONENT AND BOUNDARY SIMULATION}

To satisfy the similar requirements of the stiffening girder horizontal and vertical bending stiffness and torsional stiffness, slotted "U" - shaped spine beam is selected. Considering the bending of the main girder plane, the box beam of rectangular section is made of aluminum alloy. The stiffening girder is fixed on the box beam, whose coat is made of ABS plate. In order to meet a constant load mass and inertia moment requirements, the iron is placed in the outer side of the coat for counterweight. The deck system, such as the central separation belt, the edge of the crash barrier and the sidewalk railing are simulated. Because the torsion stiffness and torsional frequency of the arch rib are very high, the torsional stiffness of the elastic model is not considered. The bending stiffness of the arch rib is simulated by the rectangular section aluminum core beam, the aerodynamic shape is simulated by ABS plate, and mass distribution is simulated by core beam, coat and counterweight, as shown in Fig. 2.

When designing the stay cable, the similarity of the mass and the stiffness is considered strictly. The stiffness of stay cable is simulated by constantan wire and spring series, the distribution quality 
of stay cable is simulated by constantan wire, spring and plastic casing, and the frontal area of the cable surface is simulated by bushing. The drag coefficient of stay cable is 0.8 under design wind speed, which is 1.2 when the diameter of the model cable is in the millimeter range, then the external diameter of the cable is equivalent calculated in the $1.1 \sim 1.4 \mathrm{~mm}$ range. In order to avoid the obvious error, the plastic casing whose outer diameter is $1.5 \mathrm{~mm}$ is selected when the cable selection, as shown in Fig. 3.

Arch rib and the ground are consolidated. The beam is basically similar to the state, vertical, horizontal and longitudinal freedom of whose end in western side, and vertical and horizontal freedom of whose end in eastern side.

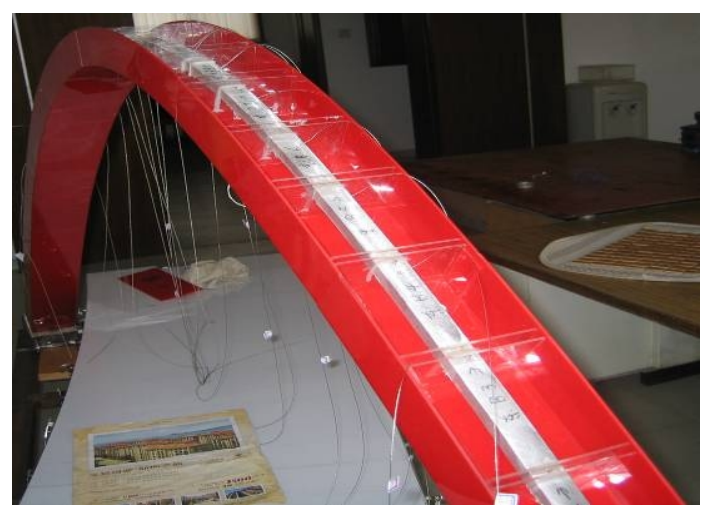

Figure 2 Arch rib core beam and coat

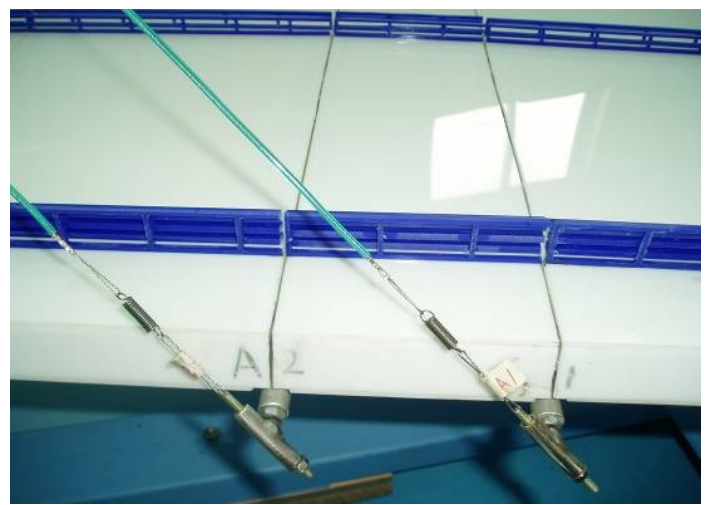

Figure 3 Cable model

\section{DYNAMIC CHARACTERISTIC ANALYSIS}

The dynamic characteristics of the structure is the basis for analysis of wind-induced vibration of bridge, of which Tongtai bridge in bare arch state and bridge state are calculated, considering the boundary condition that arch angle is embedded in the pile cap and girder and pier are simply supported. Among them, the main beam, arch rib, bridge piers are simulated by BEAM4 element, and the two phase is simulated by MASS21 element. The first order modal shapes of the bare arch and the bridge model are shown in Fig. 4 and Fig. 5.

In order to study the dynamic characteristics of the aeroelastic model, the modal test and parameter identification of the model are carried out. The error of the target frequency and the measured frequency and the vibration characteristics of the first four orders are shown in Table 2.

It can be seen that the first four orders of the model of the bare arch and the arch bridge are in good agreement with the target frequency, and the deviation is within $5 \%{ }^{[8]}$,conforming to the specification. Because the elastic modulus of the arch rib is less than the design value, the machining error exists, the arch foot can not meet the requirements of the ideal fully consolidated boundary conditions and so on, the first four orders of the model of the bare arch and the arch bridge are lower than the target frequency.

In addition, based on the random response data in the turbulent flow field, the frequency domain identification method ${ }^{[9]}$ is used to calculate the modal damping ratios of the bare arch and the state 
of the bridge. Under the bare arch, the modal damping ratio of each mode is about $0.5 \%$; under the condition of the bridge, the modal damping ratio of each mode is in the range of $1 \%-1.7 \%$. Visibly, because of the material damping of girder and sling and the friction damping between pier and girder, the modal damping ratio of each mode is higher than that of the bare arch, and the ideal damping ratio is higher than $1 \%$.

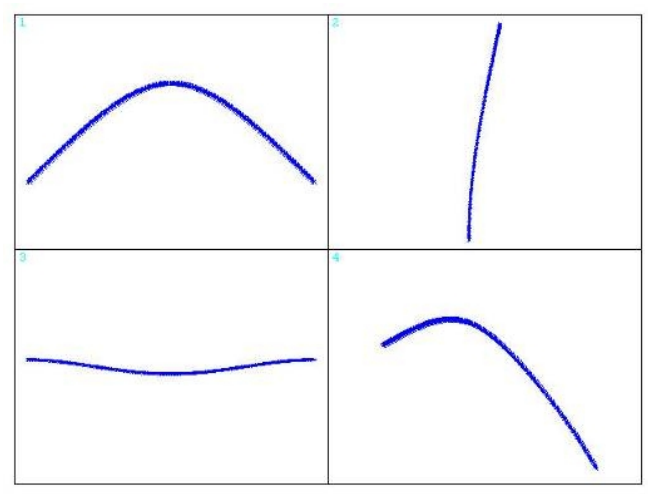

Figure 4 First order modal of bare arch structure

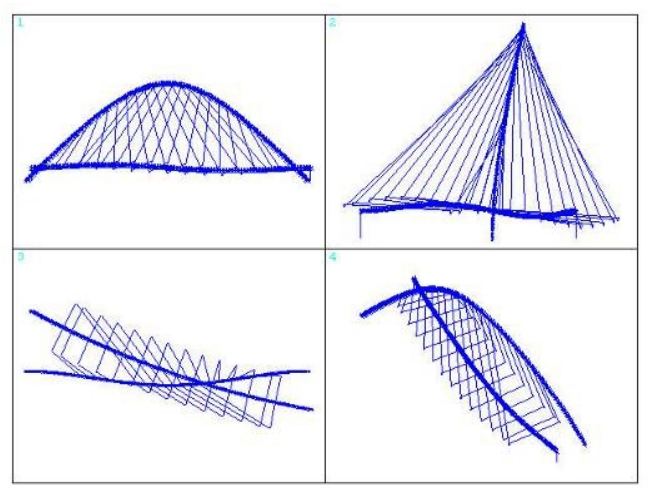

Figure 5 First order modal of bridge structure

Table 2. Analysis results of dynamic characteristics of bare arch state structure

\begin{tabular}{|c|c|c|c|c|c|c|}
\hline \multirow[t]{2}{*}{ Num } & \multicolumn{3}{|c|}{ Bare arch } & \multicolumn{3}{|c|}{ Bare arch } \\
\hline & Frequency $(\mathrm{Hz})$ & Vibration characteristics & Error(\%) & Frequency $(\mathrm{Hz})$ & Vibration characteristics & Error $(\%)$ \\
\hline 1 & 0.614 & $\begin{array}{l}\text { The first-order symmetric } \\
\text { lateral bending vibration mode }\end{array}$ & -0.60 & 0.860 & $\begin{array}{l}\text { Arch lateral bending, } \\
\text { torsion of beam }\end{array}$ & -2.19 \\
\hline 2 & 1.233 & $\begin{array}{l}\text { The first-order anti symmetric } \\
\text { vertical bending vibration mode }\end{array}$ & -1.00 & 1.130 & $\begin{array}{l}\text { Arch anti symmetric vertical } \\
\text { bending, torsion of beam }\end{array}$ & -2.57 \\
\hline 3 & 1.977 & $\begin{array}{l}\text { The first-order anti symmetric } \\
\text { lateral bending vibration mode }\end{array}$ & -1.93 & 1.249 & $\begin{array}{l}\text { Symmetrical vertical bending } \\
\text { of main beam }\end{array}$ & -3.26 \\
\hline 4 & 2.531 & $\begin{array}{l}\text { The first-order symmetric } \\
\text { vertical bending vibration mode }\end{array}$ & -2.19 & 1.727 & $\begin{array}{l}\text { Beam anti symmetric vertical } \\
\text { bending }\end{array}$ & -2.86 \\
\hline
\end{tabular}

\section{WIND TUNNEL TEST}

Tongtai bridge is in category B standard landscape site, where wind velocity changes with the height and the exponential law, index $\alpha=0.16$.Design reference wind speed is $32.5 \mathrm{~m} / \mathrm{s}$, flutter test wind speed is $52 \mathrm{~m} / \mathrm{s}$, and the critical wind velocity of static torsional divergence is $65 \mathrm{~m} / \mathrm{s}$. The results 
show that the turbulent characteristic simulation is more important than the Reynolds number in the strong turbulent flow field in the atmospheric boundary layer ${ }^{[10]}$. The wind characteristics of the atmospheric boundary layer are simulated according to the characteristics of the terrain at the bridge site, and the turbulence intensity profile as shown in Fig. 6, in which the target value of wind resistant design code for highway bridges is presented. Fig. 7 shows the wind power spectrum of simulated wind field, which simulated the wind tunnel where the height is $40 \mathrm{~cm}$, and the corresponding wind speed is $10 \mathrm{~m} / \mathrm{s}$. At the same time, the Simiu spectrum is given. Most of the energy spectrum of the simulated wind spectrum is concentrated at the low frequency end, including the main frequency of the whole bridge model which is concerned in this experiment.

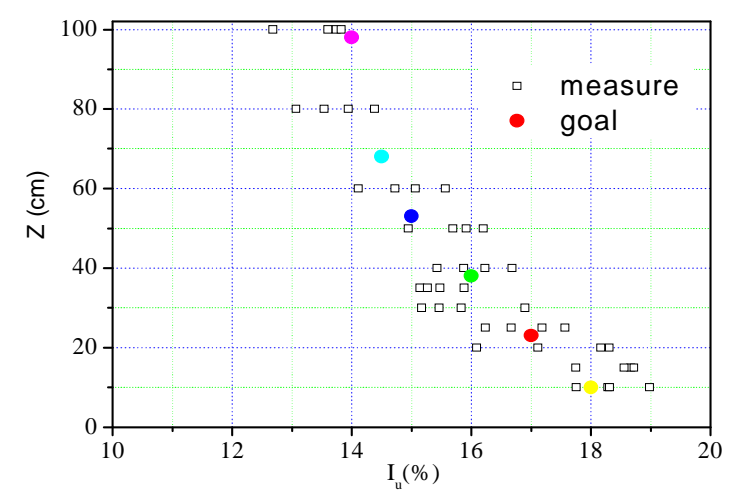

Figure 6 The simulated wind field turbulence profile

\section{UNIFORM FLOW FIELD TEST ANALYSIS}

For the wind tunnel test of the aeroelastic model, the main data collected are the vertical and lateral wind load response of the main beam and the skew arch. The laser displacement meter is installed in the main girder span, the 1/4 cross section, the arch crown and the 1/4 section of the arch rib. In the experimental process, the uniform flow field test for the bridge is found to be stable. Fig. 8 shows the change of results of the real bridge with the wind speed change, which is converted from the vertical displacement standard deviation and average value of arch crown section, under bare arch state 0 degree angle, 0 degrees and +3 degrees angle of attack condition.

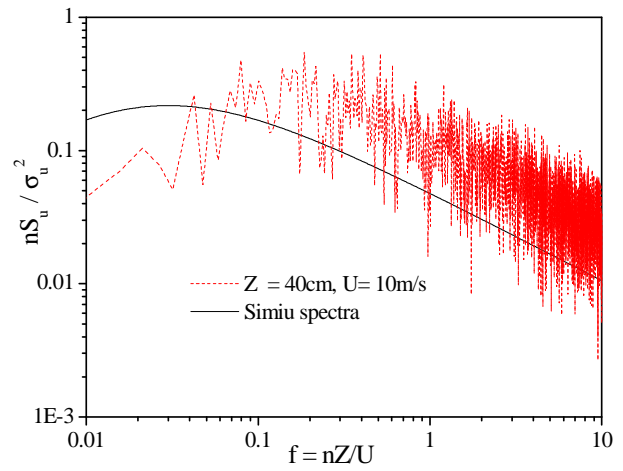

Figure 7 simulation of wind field fluctuation wind power spectrum 


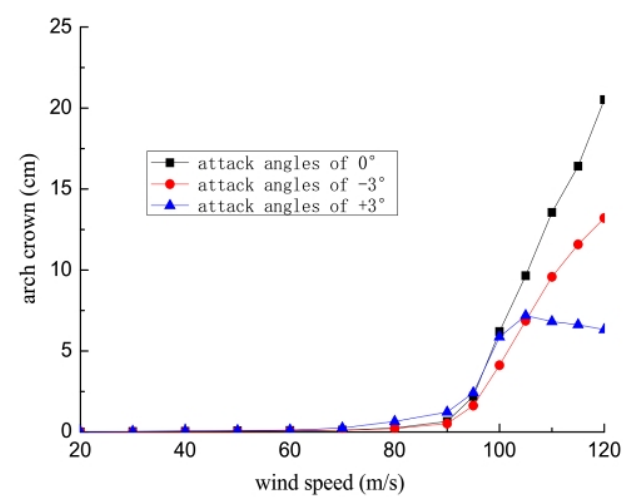

(a) Vertical displacement standard deviation

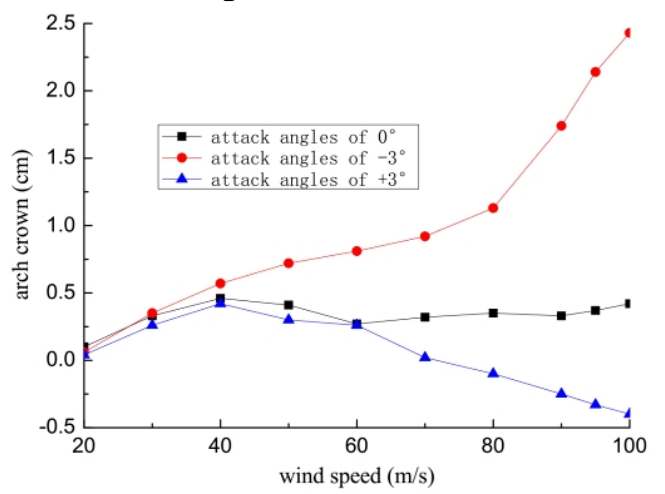

(b) Mean value of vertical displacement

Figure 8 Vertical displacement standard deviation and mean value of arch crown under different angles of attack

As shown in Fig.8(a), when the wind speed is greater than $90 \mathrm{~m} / \mathrm{s}$, the standard deviation of the vertical displacement of the arch crown section under the three conditions increases with the wind speed increasing, and there is a first order symmetric vertical bending vortex at the bare arch. And the trend can be seen from the graph that when the angle of attack is 0 , the first order symmetric vertical bending vortex vibration of the bare arch is widest, and the maximum eddy amplitude of the vault is about $20 \mathrm{~cm}$; when the angle of attack is -3 , the maximum eddy amplitude is about $14 \mathrm{~cm}$; when the angle of attack is +3 , wind speed locked area is narrowest, and the maximum eddy amplitude is about $7 \mathrm{~cm}$.

As shown in Fig.8(b), when the wind speed is greater than $80 \mathrm{~m} / \mathrm{s}$, mean value of vertical displacement of the 1/4 section of arch rib increases sharply with wind speed increases, which indicating that the bare arch has been in the static instability or close to the critical state of the static instability. Under the condition of 0 degree angle of attack and +3 degree angle of attack, the vertical displacement of arch crown section has no obvious change with the increase of wind speed, so it is not stable under the two conditions.

When the declination is 0 degrees and attack angle is 0 degree, the vortex vibration critical wind speed is about $78 \mathrm{~m} / \mathrm{s}$; When the declination is 0 degree and attack angle is plus or minus 3 degrees, vortex vibration critical wind speed is about $93 \mathrm{~m} / \mathrm{s}$ and $90 \mathrm{~m} / \mathrm{s}$, which are much higher than the design speed.

Fig. 9 shows the change of results of the real bridge with the wind speed change, which is converted from the vertical displacement standard deviation and average value of arch rib 1/4 section, for different conditions that attack angle is 0 degree and angle is different. 


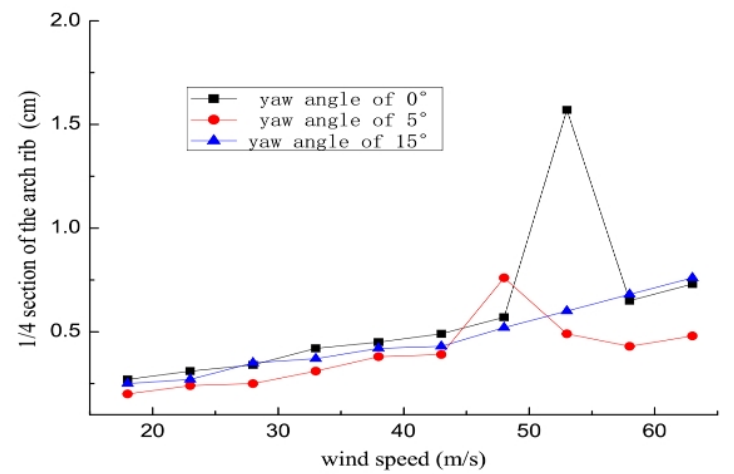

(a) Vertical displacement standard deviation

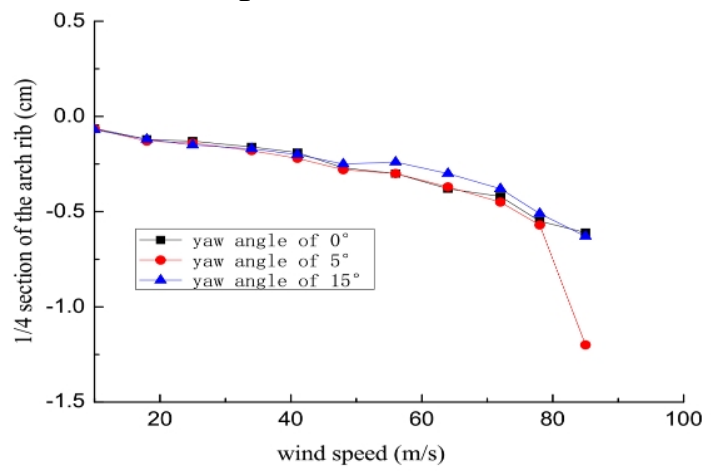

(b) Mean value of vertical displacement

Figure 9 Change of the standard deviation of the vertical displacement and the mean value of the arch rib $1 / 4$ section with wind speed at different attack angles

As shown in Fig.9(a), when angle is 0 degree and wind speed is greater than $50 \mathrm{~m} / \mathrm{s}$, vertical displacement standard deviation of $1 / 4$ cross section of arch rib changes sharply with wind speed changing, and a first order anti symmetric vertical bending vortex is appeared in the bare arch. Wind speed locking area is $48 \mathrm{~m} / \mathrm{s}-57 \mathrm{~m} / \mathrm{s}$, and the maximum vault vortex amplitude value is about $1.6 \mathrm{~cm}$; When declination is 5 degree and the wind speed is greater than $43 \mathrm{~m} / \mathrm{s}$, vertical displacement standard deviation of 1/4 cross section of arch rib changes sharply with wind speed changing, and a first order anti symmetric vertical bending vortex is appeared in the bare arch. Wind speed locking area is $43 \mathrm{~m} / \mathrm{s}-53 \mathrm{~m} / \mathrm{s}$, the maximum vault vortex amplitude value is about $0.8 \mathrm{~cm}$; Obvious changes have not occurred under 15 degree declination, that under this condition the naked arch did not occur vortex vibration phenomenon.

As shown in Fig.9(b), when declination is 5 degree and wind speed is greater than $80 \mathrm{~m} / \mathrm{s}$, the average vertical displacement with wind speed increased sharply, which indicates this naked arch has static instability or close to the static buckling critical state. When declination is 0 degree and under 15 degree, mean vertical displacement of $1 / 4$ section of arch rib increases with wind speed, which did not show significant changes, so there is no instability of the inclined arch under the two conditions.

It can be found in Fig. 8 and Fig.9 that the first order anti symmetric vertical bending vibration amplitude is very small, and the wind speed locked area is narrow; the first order symmetric vertical bending vortex amplitude is relatively large, and the wind speed lock is very wide. However, locked wind speed is higher than the design wind speed, that won't happen in reality basically. Under the three attack and a variety of angles and the wind speed is below $81.2 \mathrm{~m} / \mathrm{s}$, the bridge will not happen aerodynamic instability, and critical wind speed is higher than flutter test wind speed and static torsional divergence wind speed.

\section{EXPERIMENTAL ANALYSIS OF TURBULENT FLOW FIELD}

The mean values of the bare arch and the bridge, the standard deviation and the maximum response of the wind speed under the $65 \mathrm{~m} / \mathrm{s}$ are listed in table 3 and table 4 . Due to the test error and other 
reasons, the maximum value provided in the table is not necessarily the most unfavorable conditions, there may be slightly smaller than its corresponding operating conditions are the most unfavorable.

Table 3 The average, standard deviation and maximum response of bare arch $(\mathrm{cm})$ in the bare arch when the wind speed is $65 \mathrm{~m} / \mathrm{s}$

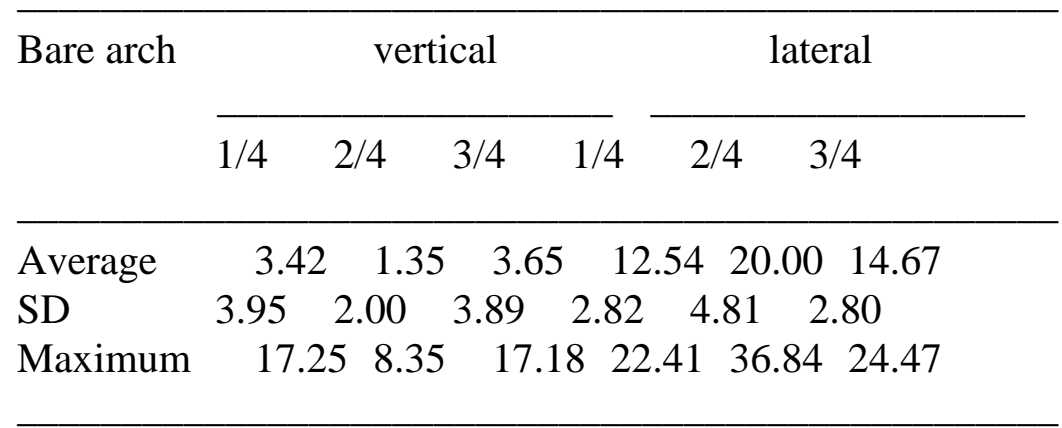

Table 3 shows that the average vertical displacement and the standard deviation of the arch crown are less than that of 1/4 section under bare arch state, and skew symmetric vertical bending stiffness and modal frequency of arch rib are lower than symmetrical vertical bending is the main reason, The average vertical displacement in arch rib elevation is lower than the lateral displacement, which shows that the lateral stiffness of the bare arch is lower than that of the vertical; The vertical displacement standard deviation in all arch rib sections is larger than average, which shows that the influence of fluctuating wind on the arch rib in the natural flow field is obvious.

Table 4 The average, standard deviation and maximum response of $65 \mathrm{~m} / \mathrm{s}$ wind velocity in turbulent flow field

\begin{tabular}{|c|c|c|c|}
\hline Bridge & mid-span & Cross-quarter & vault \\
\hline
\end{tabular}

\begin{tabular}{lcccccc}
\hline Average & 3.46 & 0.14 & 3.68 & 0.12 & 13.74 & 10.35 \\
SD & 2.71 & 0.06 & 2.51 & 0.05 & 4.36 & 3.12 \\
Maximum & 12.95 & 0.35 & 12.47 & 0.30 & 29.00 & 21.27
\end{tabular}

Table 4 shows that the mean values of vertical and torsional displacements of the bridge's main beam are small, which is secure enough; arch rib lateral displacement is relatively large, but values of each section are less than that in bare arch state, indicating that a portion of the wind load by a sling by the girder transfer to the pier, so that the arch rib lateral stiffness is improved.

In $65 \mathrm{~m} / \mathrm{s}$ natural flow field of bridge deck height, the vertical displacement of cross and 1/4 cross section of is the bridge's main beam about $13 \mathrm{~cm}$, the torsional displacement is about 0.35 degrees, and the maximum lateral displacement of the rib arch is about $30 \mathrm{~cm}$, which can be considered to have sufficient security. There is no obvious phenomenon of vortex induced vibration in the structure, under the turbulent flow field test under different incidence angles and different angle.

\section{CONCLUSIONS}

Through the wind tunnel test of Tongtai bridge, conclusion as follow:

1. The results of dynamic characteristic test of bare arch and bridge show that due to the arch rib sandwich beam material elastic modulus actual value is less than the design value, processing errors, arch foot can not meet the ideal fully consolidated boundary conditions and other reasons, the 
actual value of the model and the stiffness and frequency with respect to the target value is low, but deviation are within 5\%, which meet the requirements of the specification. The simulation result of the bare arch state is more ideal, but the full bridge modal damping is slightly higher.

2.In the uniform flow field, the first order anti symmetric vertical bending vibration amplitude is small, and the wind speed locking region is narrow; the first order symmetric vertical bending vortex vibration frequency is very high, the amplitude is very large, and the wind speed locked area is very wide. However, the critical wind speed of vortex induced vibration is higher than that of design wind speed under two conditions.

3.In turbulent flow field, the mean and standard deviation of the vertical displacement of the arch rib vault are less than that of the 1/4 section, which indicates that the lateral stiffness of the bare arch is lower than the vertical stiffness. The numerical value of the lateral displacement of the arch rib in the bridge is smaller than that of the bare arch, indicating that part of the wind load by a sling by the girder transfer to the pier, so that the arch rib lateral stiffness is improved.

4.When wind speed is lower than $81.2 \mathrm{~m} / \mathrm{s}$, the bridge is not aerodynamic instability under different angle and attack angle. In $65 \mathrm{~m} / \mathrm{s}$ natural flow field of bridge deck height, the vertical displacement of cross and $1 / 4$ cross section of is the bridge's main beam about $13 \mathrm{~cm}$, the torsional displacement is about 0.35 degrees, and the maximum lateral displacement of the rib arch is about $30 \mathrm{~cm}$, so we can think that the Tongtai bridge has enough safety.

\section{REFERENCE}

[1] Xiang Haifan. Study on bridge wind engineering into 21st century [J]. Journal of Tongji University, 2002, 30(5):529-532.(in Chinese)

[2] Zhou Dai, Shu Xinling. Wind-induced vibration and parametric analysis of single-layer reticulated shell structures [J]. Space Structure, 2003, 9(3):6 12. (inChinese)

[3] Li Yongle,Liu Wei,Xiong Wenbin,etal. Agreement Between Wind Tunnel Test and Analytical Method for the Wind-induced Response of Long span Cable-stayed Bridges[J].Engineering Mechanics, 2008, 25(8):104-108,115.

[4] Zhang Hongjie,Zhu Ledong,Hu Xiaohong.Wind Tunnel Test on Wind-resistant Stability of Super-kilometer Cable Stayed Bridge[J].China Journal of Highway and Transport.2014, 27(4):62 68.

[5] Zhao Kai, Li Yongle, Ou Yangwei, et al.Aerostatic Instability Characteristic of Long-span Cablestayed Bridges[J].Journal of Highway and Transportation Research and Development, 2011, 28(8):67 72.

[6] Emil Simiu, Robert H. Scanlan. Wind effects on structures:an introduction to wind engineering[M]. TONGJI UNIVERSITY PRESS,1992.

[7] Xiang Haifan, Lin Zhixing,Bao Weigang,et al. Wind Resistant Design Guideline for Highway Bridges[M].Beijing: China Commucations Press, 1996.

[8] Ministry of Transport of the People's Republic of China. Wind-resistent Design Specification for Highway Bridges(JTG/J-D60-01-2004). China Architecture \& Building Press, 2004

[9] Zhu Ledong. Buffeting Response of Long Span Cable-supported Bridge Under Skew Winds: Field Measurment and Analysis [D]. Hong Kong :The Hong Kong Polytechnic University, 2002.

[10] Zheng Benyou.The Preliminary Study based on the ANSYS Numerical Wind Tunnel Sirnulati[D].Beijing:Beijing Jiaotong University, 2008 